\title{
Electrodynamic influence on the diurnal behaviour of neutral daytime airglow emissions
}

\author{
Deepak K. Karan ${ }^{1,2}$, Duggirala Pallamraju ${ }^{1}$, Kedar A. Phadke ${ }^{1}$, Tatiparti Vijayalakshmi ${ }^{3}$, Tarun K. Pant ${ }^{4}$, and \\ Shyamoli Mukherjee \\ ${ }^{1}$ Physical Research Laboratory, Navrangpura, Ahmedabad, India \\ ${ }^{2}$ Indian Institute of Technology, Gandhinagar, Ahmedabad, India \\ ${ }^{3}$ Jawaharlal Nehru Technological University, Kukatpally, Hyderabad, India \\ ${ }^{4}$ Space Physics Laboratory, Trivandrum, India \\ ${ }^{5}$ Indian Institute of Geomagnetism, Navi Mumbai, India \\ Correspondence to: Duggirala Pallamraju (raju@prl.res.in)
}

Received: 11 June 2016 - Revised: 20 October 2016 - Accepted: 26 October 2016 - Published: 16 November 2016

\begin{abstract}
The diurnal variations in daytime airglow emission intensity measurements at three wavelengths OI $777.4 \mathrm{~nm}$, OI $630.0 \mathrm{~nm}$, and OI $557.7 \mathrm{~nm}$ made from a low-latitude location, Hyderabad $\left(17.5^{\circ} \mathrm{N}, 78.4^{\circ} \mathrm{E} ; 8.9^{\circ} \mathrm{N}\right.$ MLAT) in India have been investigated. The intensity patterns showed both symmetric and asymmetric behaviour in their respective diurnal emission variability with respect to local noon. The asymmetric diurnal behaviour is not expected considering the photochemical nature of the production mechanisms. The reason for this observed asymmetric diurnal behaviour has been found to be predominantly the temporal variation in the equatorial electrodynamics. The plasma that is transported across latitudes due to the action of varying electric field strengths over the magnetic equator in the daytime contributes to the asymmetric diurnal behaviour in the neutral daytime airglow emissions. Independent magnetic and radio measurements support this finding. It is also noted that this asymmetric diurnal behaviour in the neutral emission intensities has a solar cycle dependence with a greater number of days during high solar activity period showing asymmetric diurnal behaviour compared to those during a low solar activity epoch. These intensity variations over a long timescale demonstrate that the daytime neutral optical emissions are extremely sensitive to the changes in the eastward electric field over low and equatorial latitudes.
\end{abstract}

Keywords. Atmospheric composition and structure (airglow and aurora) - ionosphere (equatorial ionosphere; ionosphere-atmosphere interactions)

\section{Introduction}

It is known that optical airglow emissions act as tracers of atmospheric behaviour that exists at the altitudes of their origin. In the earth's upper atmosphere, optical emissions originate when atomic or molecular constituents or their ions de-excite from their higher energy states to the lower ones. The emissions emanate at different altitudes depending on the constituents and type of chemical/photochemical reactions that produces them at those altitudes. Understandably, the variability of the reactants that participate in these reactions has a role in the overall variability of the airglow emissions. In that regard, the Doppler shifts and widths of the neutral $630.0 \mathrm{~nm}$ nightglow emissions have been used to obtain information on thermospheric winds and neutral thermospheric temperatures. With the knowledge that dissociative recombination is responsible for the $630.0 \mathrm{~nm}$ nightglow, the all-sky images of this emission yielded unique signatures of the reversal of the equatorial ionization anomaly (EIA) in the nighttime (Sridharan et al., 1993a). The all-sky images also provide information on the dynamics of large-scale plasma bubbles (e.g. Taylor et al., 1995; Makela et al., 2013). The mesospheric $\left(\mathrm{OH}, \mathrm{O}_{2}\right.$ band) and lower thermospheric (OI $557.7 \mathrm{~nm}$ ) emission variability has been used to derive information on the mesospheric temperatures (e.g. Taylor et al., 1995; Singh and Pallamraju, 2015), atmospheric gravity waves (Shiokawa et al., 2009; Singh and Pallamraju, 2016), tides, and planetary-scale waves (Nakamura et al., 1998). 
With regard to the daytime airglow, predominantly OI $630.0 \mathrm{~nm}$ emissions have been used to derive the effect of coronal mass ejection on the thermosphere (Pallamraju and Chakrabarti, 2005), solar flares (Das et al., 2010), and magnetospheric cusps (Pallamraju et al., 2004) and to understand the upper-atmospheric behaviour due to solar activity. Similar variations between daily averaged $630.0 \mathrm{~nm}$ dayglow emission intensity and the daily sunspot number indicated the influence of solar flux on the optical dayglow emissions (Pallamraju et al., 2010). With the addition of OI $557.7 \mathrm{~nm}$ for daytime measurements, investigations have been extended to study various aspects such as gravity wave dynamics in the lower thermosphere (Laskar et al., 2013, 2015), the effect of tidal and solar flux variations on mesosphere and lower-thermosphere (MLT) dynamics from onboard the Upper Atmosphere Research Satellite (UARS; e.g. Maharaj-Sharma et al., 2004; Zhang and Shepherd, 2004, 2005), and three-dimensional daytime wave characteristics (Pallamraju et al., 2016). In all these measurements it was seen that the diurnal behaviour of daytime intensity broadly varied as a function of solar zenith angle. In fact, the empirical models for OI $557.7 \mathrm{~nm}$ and OI $630.0 \mathrm{~nm}$ dayglow emissions that were developed (Zhang and Shepherd, 2004, 2005) do have solar zenith angle as one of the inputs. This is not unexpected, as the solar photons through the processes of photodissociation, photoelectron production, and ionization at a given location do affect the volume emission rates of the daytime airglow emissions. This gives rise to a broad solar zenith-angle-dependent behaviour for the diurnal emission intensity distribution (Solomon and Abreu, 1989). Thus, one would expect that with the decreasing/increasing of the solar zenith angle, the dayglow emission intensities will increase/decrease giving rise to maximum dayglow emission intensities around local noon resulting in a symmetric pattern in the diurnal variation of emission intensities with respect to local noon. However, the high temporal resolution groundbased measurements that we have carried out do show that the diurnal behaviour of dayglow emission intensities does not follow a symmetric pattern with respect to local noon on several days. This non-symmetric or asymmetric diurnal behaviour in the intensity pattern with respect to the local noon on a given day has been investigated at greater depths in this work. The influence of neutral winds vs. that of equatorial electrodynamics on the daytime airglow production mechanisms and, thereby, the resulting emission intensities have been assessed. It is found that the electrodynamic behaviour is mainly responsible for such disparity that is seen in the diurnal behaviour of neutral dayglow emission intensities. Moreover, the extent of asymmetricity in the diurnal behaviour in the dayglow emission intensities is found to show a solar activity dependence with a greater number of days showing asymmetric diurnal behaviour during high solar activity periods.

\section{Observational technique and data analysis}

\subsection{Oxygen dayglow emissions}

Ground-based optical instruments are now available that provide opportunities to measure the thermospheric dayglow emissions buried in the strong solar background continuum. A few methods have been put forth in the past (e.g. Narayanan et al., 1989; Sridharan et al., 1993b, 1998; Chakrabarti et al., 2001; Pallamraju et al., 2002, 2013; Gerard and Meriwether, 2011) to measure these emission intensities, which used low- and high-resolution Fabry-Pérot etalons, echelle and normal gratings, with varying fields of view.

In the present work the thermospheric optical dayglow OI emission intensities measured have been obtained by using a high spectral resolution Multiwavelength Imaging Spectrograph using Echelle grating (MISE) (Pallamraju et al., 2013) from a low-latitude station, Hyderabad, India $\left(17.5^{\circ} \mathrm{N}\right.$, $78.4^{\circ} \mathrm{E} ; 8.9^{\circ} \mathrm{N}$ MLAT). MISE obtains dayglow emission intensities over a large field of view $\left(\sim 100^{\circ}\right)$ along the slit direction, simultaneously at three wavelengths: OI 557.7, OI 630.0 , and OI $777.4 \mathrm{~nm}$ (without any need to change the grating angle). Both photochemical and chemical reactions are responsible for the production of these three optical dayglow emissions. The availability of the reactants, such as photoelectrons, and atmospheric constituents responsible for an emission decide the altitudes of emissions, which are around $130 \mathrm{~km}$ (average of the two peak emission altitudes at $\sim 100$ and $\sim 160 \mathrm{~km}), 230$, and $300 \mathrm{~km}$ for the $\mathrm{O}\left({ }^{1} \mathrm{~S}\right)(557.7 \mathrm{~nm})$, $\mathrm{O}\left({ }^{1} \mathrm{D}\right)(630.0 \mathrm{~nm})$, and $\mathrm{O}\left({ }^{5} \mathrm{~S}\right)(777.4 \mathrm{~nm})$ emissions, respectively. The $\mathrm{O}\left({ }^{1} \mathrm{~S}\right)$ state is produced due to photoelectron impact on the ground state of $\mathrm{O}$, collisional deactivation of $\mathrm{N}_{2}$, photodissociation of $\mathrm{O}_{2}$ in $90-120 \mathrm{~nm}$ of the solar radiation, and dissociative recombination of $\mathrm{O}_{2}^{+}$(e.g. Tyagi and Singh, 1998; Witasse et al., 1999; Zhang and Shepherd, 2005). Also, a three-body reaction (Barth mechanism) is responsible for the production of $557.7 \mathrm{~nm}$ emissions at lower altitudes below $120 \mathrm{~km}$ (which peak at $\sim 100 \mathrm{~km}$ ). The dissociative recombination of $\mathrm{O}_{2}^{+}$, which depends on the electron densities, contributes significantly to the production of $557.7 \mathrm{~nm}$ dayglow emissions at higher altitudes, especially in low and equatorial latitudes (e.g. Tyagi and Singh, 1998; Upadhayaya et al., 2002; Taori et al., 2003). Rocket-borne O $\left({ }^{1} \mathrm{~S}\right) 557.7 \mathrm{~nm}$ measurements (Wallace and McElroy, 1966), nightglow measurements from the Wind Imaging Interferometer (WINDII; Shepherd et al., 1997), and ground-based optical dayglow measurements (Taori et al., 2003) reported a dominant F region contribution as compared to that of lower altitudes. The $630.0 \mathrm{~nm}$ is produced due to the de-excitation of $\mathrm{O}\left({ }^{1} \mathrm{D}\right)$ to $\mathrm{O}\left({ }^{3} \mathrm{P}\right)$ state. The $\mathrm{O}\left({ }^{1} \mathrm{D}\right)$ state is produced by photoelectron impact on the ground state $\mathrm{O}$, photodissociation of $\mathrm{O}_{2}$ in the Schumann-Runge continuum (135-175 nm) of the solar radiation, a dissociative recombination of $\mathrm{O}_{2}^{+}$, and a cascade from the loss of $\mathrm{O}\left({ }^{1} \mathrm{~S}\right)$, with the last one being a minor con- 
tributor (Solomon and Abreu, 1989; Witasse et al., 1999). The photoelectron impact mechanism contributes most to the $630.0 \mathrm{~nm}$ dayglow emissions followed by the photodissociation mechanism. Dissociative recombination contribution to the $630.0 \mathrm{~nm}$ dayglow emission is around $20-30 \%$ (Hays et al., 1978; Singh et al., 1996). However, the dissociative recombination contributes significantly to the temporal variability of the emissions (Sridharan et al., 1992, 1994; Pallam Raju et al., 1996). The de-excitation of $\mathrm{O}\left({ }^{5} \mathrm{~S}\right)$ to $\mathrm{O}\left({ }^{5} \mathrm{P}\right)$ yields $777.4 \mathrm{~nm}$ dayglow emissions. The $\mathrm{O}\left({ }^{5} \mathrm{~S}\right)$ state is produced due to the radiative recombination of $\mathrm{O}^{+}$and $e^{-}$(Tinsley et al., 1973). This emission maximizes at the peak of the $F$ layer where the densities of both $\mathrm{O}^{+}$and $e^{-}$are maximal.

The variation in intensities at these three optical thermospheric dayglow emissions carries the information of the dynamical behaviour occurring at the respective altitudes of emissions. The dayglow emission intensities at the three wavelengths are imaged onto a $1 \mathrm{k} \times 1 \mathrm{k}$ charge coupled device (CCD) detector to form a high-resolution $(0.012 \mathrm{~nm}$ at $630.0 \mathrm{~nm}$ ) spectral image. On-chip binning of eight pixels along the spatial direction is carried out to increase the signal-to-noise ratio. The spectra obtained from MISE are compared with the normalized standard solar spectrum in order to calibrate them in the wavelength domain. These are then compared at the continuum level to obtain the contributions of total dayglow emissions and the atmospheric scattering (Ring effect) (Pallamraju et al., 2000). The dayglow emission intensities are obtained by removing the scattering contribution with a data cadence of $5 \mathrm{~min}$. This method of retrieval of dayglow is well-established and is described in detail in the literature (Pallamraju et al., 2002, 2013). The technical details of MISE have been described by Pallamraju et al. (2013). Further, in order to make a comparison of our findings with earlier dayglow measurements, we have made use of the OI $630.0 \mathrm{~nm}$ data as presented in the published literature.

\subsection{EEJ data}

Equatorial electrojet (EEJ) refers to the intense narrow jet of current in the eastward direction that flows in the daytime over the dip equator. EEJ strength can be obtained by measuring the induced magnetic field using magnetometers placed on the surface of earth. Over Indian longitudes the horizontal component of the earth's magnetic field is obtained from two stations. One station is at Tirunelveli (TIR) $\left(8.7^{\circ} \mathrm{N}, 77.8^{\circ} \mathrm{E} ; 0.15^{\circ} \mathrm{N} \mathrm{MLAT}\right)$, in a magnetic equatorial region which records the influence of currents induced due to the EEJ, and the other is at Alibag $(\mathrm{ABG})\left(18.6^{\circ} \mathrm{N}, 72.9^{\circ} \mathrm{E}\right.$; $10.5^{\circ} \mathrm{N}$ MLAT), magnetically an off-equatorial station, that is not influenced by the EEJ currents. From each station, variations relative to their nighttime base values are subtracted so that the contribution from magnetospheric currents, if any, is removed. Thus, the difference between the magnetic data at Tirunelveli and Alibag yields information on the strength of

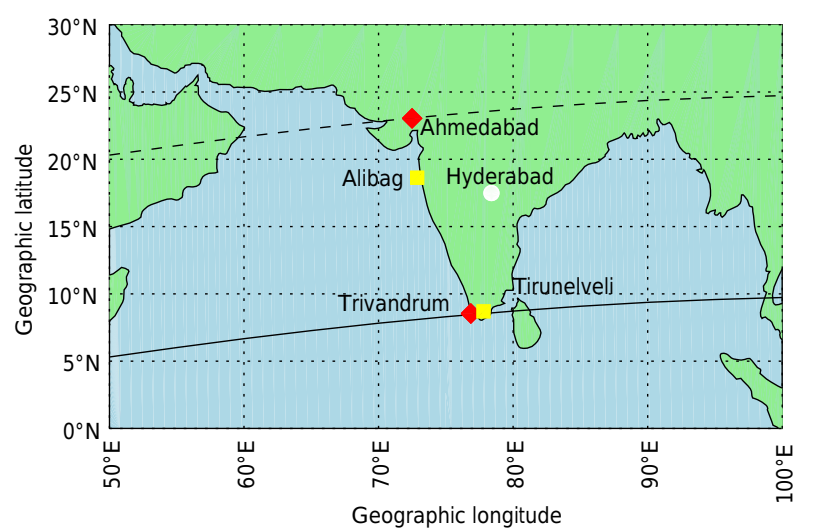

Figure 1. The geographic locations of the stations from which data have been obtained are shown. Neutral optical dayglow emission data have been obtained by MISE commissioned at Hyderabad (white dot). The ionospheric data obtained from digisondes located at Trivandrum and Ahmedabad are shown as red diamonds. Stations from which the magnetic data are obtained to calculate the EEJ strengths are marked as yellow squares. The solid and dashed dark lines represent the geomagnetic equator (obtained from IGRF12) (Emmert et al., 2010) and the EIA crest location in the Northern Hemisphere $\left(+15^{\circ}\right)$, respectively, for the year 2014 .

the EEJ current. The high temporal resolution (1 min data cadence) EEJ data from December 2013 to March 2014 have been used in the present work.

\subsection{Ionospheric data}

Ionospheric behaviour from two stations, Trivandrum $\left(8.5^{\circ} \mathrm{N}, 76.9^{\circ} \mathrm{E} ; 0.07^{\circ} \mathrm{N}\right.$ MLAT), a geomagnetically equatorial location, and Ahmedabad $\left(23.0^{\circ} \mathrm{N}, 72.5^{\circ} \mathrm{E} ; 14.9^{\circ} \mathrm{N}\right.$ MLAT), typically the northern crest location of the EIA, has been used. The data cadence for the ionospheric information is $7.5 \mathrm{~min}$.

Figure 1 shows the geophysical locations of all the stations from which the data are used for the present work. The $x$ and $y$ axes show the geographic longitude and latitude, respectively. Neutral optical dayglow emission data are obtained from the location Hyderabad, marked as a white solid circle on the map. The red diamonds and yellow squares show the stations from which ionospheric information and the EEJ data are obtained. The dark solid line represents the geomagnetic equator (obtained from the International Geomagnetic Reference Field (IGRF-12) model for 2014.0), whereas the dashed line shows the typical northern crest region $\left(15^{\circ} \mathrm{N}\right.$ MLAT) of the EIA.

In the present work the optical dayglow emission data obtained from December 2013 to March 2014 have been used to address this issue. Independent ionospheric data and the EEJ data obtained during this period have been used to substantiate our findings. 


\section{Results}

Figure 2 depicts examples of diurnal variability on 2 days for OI $630.0 \mathrm{~nm}$ dayglow emissions, with the $x$ and $y$ axes showing local time (LT) in hours and intensity in rayleighs. The black solid line shows the 11-point running average. A dotted vertical line is drawn at local noon to aid the eye in bringing out the contrast between the pre- and post-noon behaviour in the emission intensity variability. It can be readily noted that on 5 January 2014 (Fig. 2a), the intensity variability is symmetric with peak intensity around noontime, which seems almost like an inflexion point. This behaviour is contrary to that obtained on 19 December 2013 (Fig. 2b), when the peak intensity was reached in the afternoon hours. As discussed above, the photochemical production is expected to peak around noontime, and, therefore, this asymmetric diurnal behaviour in the dayglow emission intensities seems anomalous. It may also be noted that the rate of rise in intensities is different on these 2 days. The extent of asymmetry can be quantified as the product of difference in times between those of peak intensity and local noon and the ratios of the intensities at those times. Mathematically, the asymmetricity in time (AT) is given as

$\mathrm{AT}=\left(\frac{I_{\text {peak }}}{I_{\text {noon }}}\right) \times\left(T_{\text {peak }}-T_{\text {noon }}\right)$,

where $T_{\text {peak }}$ and $T_{\text {noon }}$ are the times of peak emission intensity and local noon. $I_{\text {peak }}$ and $I_{\text {noon }}$ are the intensity values corresponding to $T_{\text {peak }}$ and $T_{\text {noon }}$, respectively. If the peak intensity occurs in pre-noon hours, it can be seen from Eq. (1) that the AT becomes negative. The diurnal emission intensity pattern is considered to be symmetric or asymmetric for AT $\leq 0.4 \mathrm{~h}$. and AT $>0.4 \mathrm{~h}$, respectively. The AT values for 5 January 2014 and 19 December 2013 are calculated to be $0.4 \mathrm{~h}$ (symmetric diurnal behaviour) and $1.1 \mathrm{~h}$ (asymmetric diurnal behaviour), respectively.

Such behaviour in the diurnal intensity pattern is seen not only in the OI $630.0 \mathrm{~nm}$ emissions but also in the emissions at OI $777.4 \mathrm{~nm}$ and OI $557.7 \mathrm{~nm}$ that emanate from altitudes above and below that of the OI $630.0 \mathrm{~nm}$ dayglow. Figure 3 shows the diurnal intensity behaviour, according to which the $x$ axes represent the local time and the $y$ axes show the intensity of the optical emissions. The vertical dotted line represents the local noontime. All the data included here correspond to magnetically quiet days $(\mathrm{Ap}<23)$. The total number of days plotted in each panel may be noted on the top right corner of each figure. The upper (Fig. 3a, d), middle (Fig. 3b, e), and lower (Fig. 3c, f) panels show the diurnal behaviour of dayglow emission intensities at 777.4, 630.0, and $557.7 \mathrm{~nm}$ wavelengths, respectively. Figure $3 \mathrm{a}-\mathrm{c}$ and $\mathrm{d}-$ $\mathrm{f}$ show the behaviour on several days when the diurnal intensity pattern was symmetric (AT $\leq 0.4 \mathrm{~h})$ and asymmetric (AT $>0.4 \mathrm{~h}$ ), respectively. Note the difference in the timings of the occurrence peaks with respect to local noon, which are different for different days. The yellow line shows the aver- age of all the days of data, which is essentially drawn to show the contrasting diurnal behaviour in each emission. The difference in the pattern of the emission intensities on the days with symmetric/asymmetric diurnal behaviour is clearly contrasting in many ways. (1) It may be noted that the intensity variability in the days with symmetric diurnal behaviour is not as much as that seen in the days with asymmetric diurnal behaviour. (2) The post-noon spread in intensities on the days with asymmetric diurnal behaviour is much greater as compared with that in the pre-noon. (3) The pattern of rise in intensity is different between these two types, with a slow rate of rise on the days with asymmetric diurnal behaviour, while it is relatively faster on the days that show symmetric diurnal behaviour. (4) While the emission intensity pattern seems skewed towards post-noon on the days with asymmetric diurnal behaviour, it seems slightly skewed towards forenoon, especially in the $630.0 \mathrm{~nm}$ and OI $557.7 \mathrm{~nm}$ emissions, on the days with symmetric diurnal behaviour. In Fig. 3 we collated the days with symmetric/asymmetric diurnal behaviour in emission intensities at each of the wavelengths, obtained from December 2013 to March 2014. There are days when only one or two emission intensities show symmetric diurnal behaviour but the others show asymmetric behaviour. There are also common days when all the oxygen emission intensities show symmetric/asymmetric diurnal behaviour. All these features make this very interesting and intriguing at the same time, as there seems to be a combination of atmospheric processes, the constituents/reactants available at a given time, neutral dynamics (winds), and electrodynamic forces operative at different altitudes on these days.

To begin with, let us look at the production mechanisms for these emissions as discussed in the earlier section. It is clear that the 557.7 and $630.0 \mathrm{~nm}$ dayglow emissions depend on both neutral and electron densities, whereas the $777.4 \mathrm{~nm}$ emissions depend only on the ion and electron densities. Thus, the production of all three dayglow emission intensities depends on the available solar flux, temperaturedependent reaction rates, and densities of neutrals and ions. It can be readily seen that photoelectrons, extreme ultraviolet (EUV) flux for photodissociation, and ionization at any location vary with respect to the solar zenith angle. So the diurnal pattern of the three dayglow emission intensities is expected to be symmetric with respect to local noontime. Thus, the asymmetric diurnal behaviour observed in the three optical dayglow emission intensities (Fig. 3) can mainly be due to the variation of either the neutral densities or the electron densities, which can be engendered by neutral dynamics or electrodynamics or both.

Firstly, we consider the changes in electron densities due to winds, especially the meridional wind, as it is known that the wind-assisted movement of electrons along the magnetic field lines does alter the electron densities at a given location. An increase/decrease of the electron number densities results in a corresponding increase/decrease in the optical emission intensities. A poleward wind moves the ionospheric layer to 

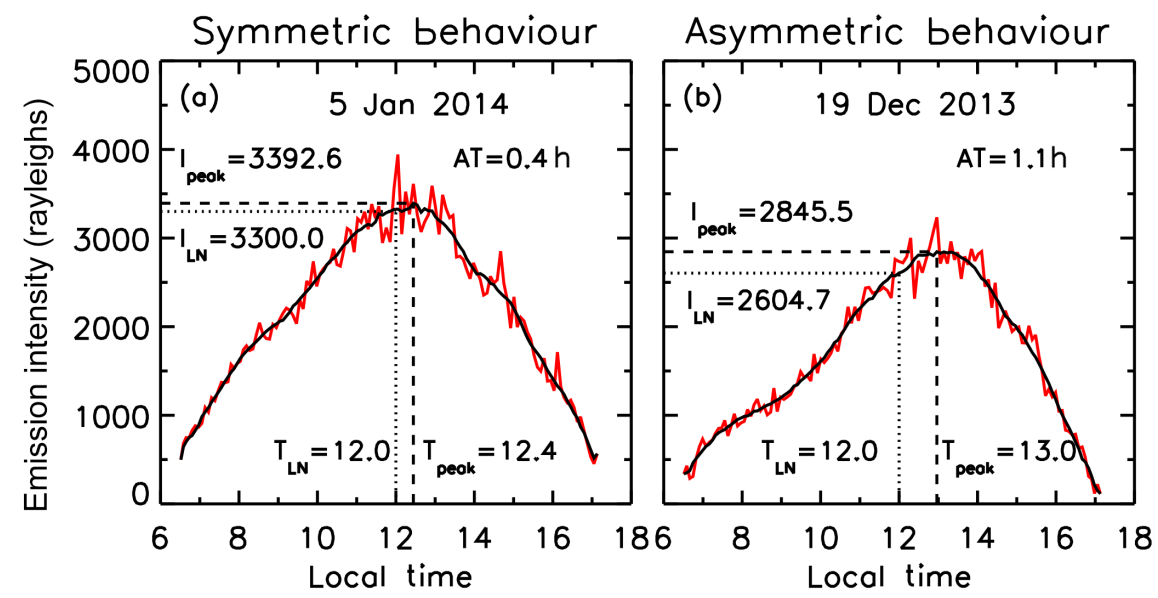

Figure 2. Sample of the diurnal behaviour of OI $630.0 \mathrm{~nm}$ dayglow emissions. (a) Symmetric diurnal behaviour in intensities with respect to local noon. The behaviour shows solar zenith-angle-dependent variation. Note that with respect to noon the rise and fall in intensities seem symmetric. (b) Asymmetric diurnal behaviour in intensities with respect to local noon. The peak in intensities is achieved after about $1 \mathrm{~h}$ from noon. Note the rise in intensities is more gradual than the decrease. The product of ratio of intensities at the peak to those at noon and the difference in times between the peak reached and local noon yield the value of asymmetricity in time (AT), which is also shown in both the panels.

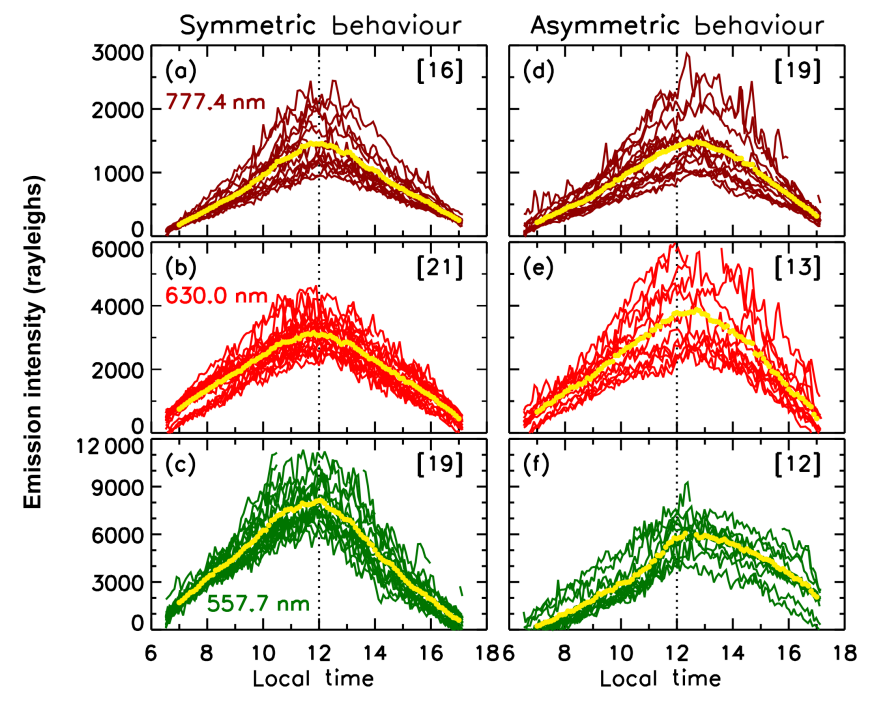

Figure 3. The diurnal behaviour in optical dayglow emission intensities at (a, d) OI $777.4 \mathrm{~nm}$, (b, e) OI $630.0 \mathrm{~nm}$, and $(\mathbf{c}, \mathbf{f})$ OI $557.7 \mathrm{~nm}$ is shown. The plots in the left column (a, b, c) show the plots with symmetric diurnal behaviour in intensities with respect to local noon. Plots (d, e, f) show asymmetric diurnal behaviour in intensities with respect to local noon. The yellow line shows the average of all the days of the data. The number of days of data that exist for a given diurnal behaviour is shown in square brackets.

lower altitudes where the dissociative recombination mechanism can be significant, thereby increasing the yield of OI $630.0 \mathrm{~nm}$ and OI $557.7 \mathrm{~nm}$ dayglow emissions. Similarly, the yield of the OI $777.4 \mathrm{~nm}$ dayglow emission is also expected to show an enhancement through the radiative recombination mechanism. An equatorward wind will move the iono- spheric layer to higher altitudes, thereby reducing the potential yield of the dayglow emissions. In a similar manner, poleward winds, especially from the winter hemisphere, bring additional plasma into the summer hemisphere and so give rise to a greater yield in all the daytime airglow emissions.

Other than meridional winds, the equatorial electrodynamic forcing is another potential cause which is capable of bringing plasma to low latitudes. In the equatorial region, due to the horizontal nature of the magnetic field lines, several interesting phenomena take place. In the daytime, the formation of the equatorial electrojet is one of them, the strength of which can be used to infer the effectiveness of the electrodynamic processes operative on that day in the low latitudes. Under the action of the equatorial $F$ region electric fields and the northward directed magnetic field lines of the earth, the plasma undergoes an $\boldsymbol{E} \times \boldsymbol{B}$ drift, which moves it to higher altitudes, from where the plasma follows the magnetic field lines and is transported to latitudes farther away due to the pressure gradient forces. This action forms the well-known EIA. Such excess ionization that is brought in to a given off-equatorial location results in greater emission intensities through the dissociative recombination and radiative recombination mechanisms. Moreover, in off-equatorial low-latitude regions, the westward zonal wind can produce an eastward $\mathrm{E}$ region electric field which gets mapped to the equatorial $F$ region through magnetic field lines and can contribute to the $\boldsymbol{E} \times \boldsymbol{B}$ plasma drifts, thereby increasing the EIA strength. Thus, the effect of the zonal winds on the optical dayglow emission intensities can also be brought about through the equatorial electrodynamics. The relative importance of these two sources, namely neutral winds and electrodynamics, in making the diurnal emission behaviour to be 
asymmetric in the dayglow emission intensities is evaluated below.

Figure 4 shows 2 individual days (columns) of data for all the OI emissions, thermospheric neutral winds, and EEJ strengths (rows). The left column shows the day with a symmetric diurnal pattern (26 December 2013), and the one on the right shows the day with an asymmetric diurnal pattern (7 February 2014) in all the dayglow emission intensities. The $x$ axes show local time, while the $y$ axes (in the top three rows) show the dayglow intensity and (fourth row) the thermospheric zonal wind, $U_{x}$ (dotted line, positive eastward), and meridional wind, $U_{y}$ (solid line, positive northward), at all three emission altitudes on both the days as obtained by the Horizontal Wind Model (HWM14) (Drob et al., 2015). The bottommost row shows EEJ strength in nanotesla, and the horizontal dashed line drawn corresponds to $0 \mathrm{nT}$ value. The solid lines in the dayglow emission intensities represent an 11-point running average of the data. The vertical dotted lines are drawn at local noontime. The dates of data and the values of AT of each of the emissions are shown in the plots. For these days, panels $\mathrm{d}$ and $\mathrm{i}$ show the zonal and meridional wind at 130,230 , and $300 \mathrm{~km}$ altitudes from where the OI $557.7 \mathrm{~nm}$, OI $630.0 \mathrm{~nm}$, and OI $777.4 \mathrm{~nm}$ emissions originate. It is expected that larger meridional winds bring the ionospheric layer to lower altitudes, which give rise to larger dayglow emissions, as discussed above. The meridional wind magnitudes were large on 26 December 2013 at all the altitudes, which, expectedly, are favourable for giving rise to an asymmetric diurnal pattern in emission intensities; however, the observations do not show such a behaviour. Conversely, on the day (7 February 2014) with lower meridional wind magnitudes, asymmetric diurnal behaviour was observed in all the dayglow emission intensities. Therefore, the meridional wind hypothesis as the cause of the asymmetric diurnal behaviour is not supported by observations.

In this context, the possibility of electrodynamic influence in bringing about the asymmetric diurnal behaviour in the intensities is examined using the equatorial electrojet strength as the reference. It is known that the EEJ dynamics have a significant role to play not only in influencing the distribution of electron densities in low-latitude regions (Moffett, 1979) but also in influencing the zonal winds and temperatures (Raghavarao et al., 1993). It should be noted that the peak EEJ strengths on these 2 days were different (40 and $75 \mathrm{nT}$ ); however, the peak emission intensities on these days were similar, and therefore it is apparent that the peak EEJ strengths have no direct relationship with the magnitudes of the peak dayglow intensities. However, it has been shown in earlier works (Raghavarao et al., 1978) that the integrated EEJ strength until noontime has a direct one-to-one correlation with the strength of the EIA. Although ionization is not measured through optical measurements, it has been shown by earlier studies that the ionization brought in from equatorial latitudes contributes to the OI $630.0 \mathrm{~nm}$ dayglow emissions through a dissociative recombination mechanism (Srid-

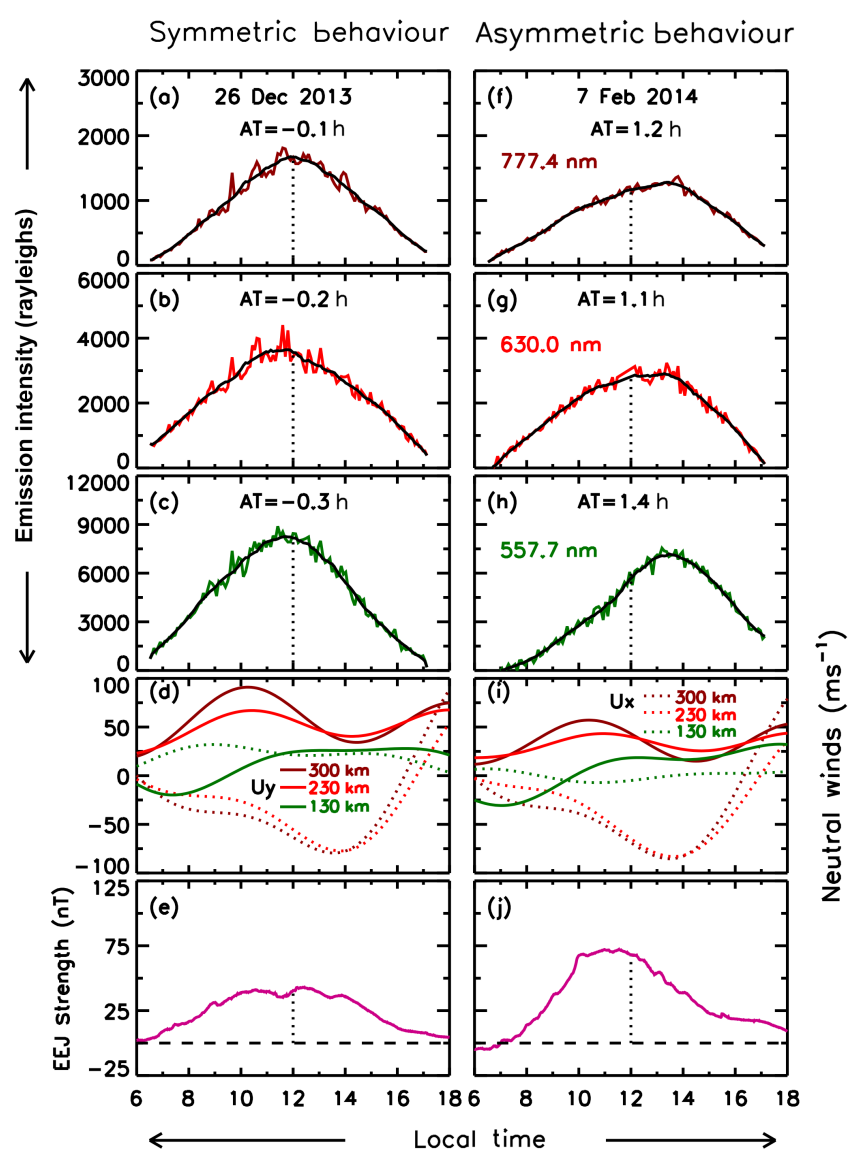

Figure 4. Top row (a,f) shows the diurnal variability in OI $777.4 \mathrm{~nm}$ on 2 selected days. Plots $(\mathbf{b}, \mathbf{g})$ and $(\mathbf{c}, \mathbf{h})$ show the diurnal behaviour of OI $630.0 \mathrm{~nm}$ and OI $557.7 \mathrm{~nm}$ emissions on the same days as of OI $777.4 \mathrm{~nm}$ emissions. Plots (d, i) show the HWM14 neutral wind magnitudes. Plots (e, j) show the electrojet strengths. The plots in the left column show the symmetric diurnal behaviour in all three oxygen dayglow emission intensities, while those on the right represent the day with asymmetric diurnal behaviour in all the dayglow emission intensities.

haran et al., 1992; Pallam Raju et al., 1996; Pallamraju et al., 2002). Thus, the asymmetricity in time observed in optical measurements at all the emission wavelengths is compared with the values of the EEJ strengths $\left(A_{\mathrm{EEJ}}\right)$ integrated over 07:00-12:00 LT. On these days with symmetric and asymmetric diurnal patterns in dayglow emission intensities, the values of $A_{\text {EEJ }}$ were 147 and $214 \mathrm{nT}$ h. As discussed above in this section, the larger value of $A_{\mathrm{EEJ}}$ on the day with asymmetric diurnal behaviour enhances the strength of the EIA and results in higher values of AT. The values of AT on the day with asymmetric diurnal behaviour were calculated to be $1.2,1.1$, and $1.4 \mathrm{~h}$ for $777.4,630.0$, and $557.7 \mathrm{~nm}$ emissions, respectively, whereas on the day with symmetric diurnal behaviour, these values were $-0.1,-0.2$, and $-0.3 \mathrm{~h}$ for these emissions. The zonal wind magnitudes show nearly similar behaviour on both these days. In any case, the zonal winds 
affect the equatorial electrodynamics, and, therefore, their effect will be implicit in the integrated EEJ strength.

Figure 5 comprehensively summarizes the results of the present investigation. The $x$ axis shows the day number beginning on 1 December 2013. Figure 5a shows the peak meridional wind, $U_{y}$ (solid line), and the corresponding zonal wind, $U_{x}$ (dotted line), magnitudes obtained from the HWM14 model (Drob et al., 2015) for the three emission altitudes. It can be seen that the meridional wind reduces in magnitude, expectedly, from December to March, which is consistent with the seasonal wind behaviour as one moves from the solstice to the equinox, whereas the variation in zonal winds is not significant. Figure $5 \mathrm{~b}$ shows the AT values for all these three emissions, which over this period varied from -1.5 to $+1.5 \mathrm{~h}$. From the uncorrelated behaviour between the values of AT and the meridional winds, it is clear that meridional winds are not the cause of the observed asymmetric diurnal behaviour in the optical emission intensities. Figure $5 \mathrm{c}$ shows the EEJ strengths, $A_{\mathrm{EEJ}}$, integrated in the pre-noon hours (07:00-12:00 LT), which display a similar behaviour to that in the AT values in the optical dayglow emission intensities at all three wavelengths. The optical dayglow emissions are affected by both neutral dynamics and electrodynamics; both of these show seasonal dependence. So their contributions in the observed dayglow fluctuations are different in different seasons. The strength of equatorial electrodynamics decides the latitudinal extent of the EIA. Also, the effect of the EIA can first be seen at lower altitudes in off-equatorial latitudes and then at higher altitudes. Thus, on a given day this can result in better agreement between $A_{\mathrm{EEJ}}$ and values of AT in $557.7 \mathrm{~nm}$ emission intensity but not in 630.0/777.4 nm emissions. In Fig. 5, AT values are calculated for all the clear-sky days irrespective of symmetric or asymmetric diurnal patterns at all three emission wavelengths, which shows a broad similarity with the values of $A_{\mathrm{EEJ}}$ at different wavelengths of emissions. This clearly indicates that electrodynamic variations primarily govern the diurnal behaviour of the neutral dayglow emission intensities.

For the sake of completeness, it should however be mentioned that the thermospheric winds used in this study are model driven, whereas the EEJ values were obtained from measurements. Model values of winds have been used as measurements of winds in the daytime are not available for comparison. Nevertheless, as they are driven primarily by solar heating, it is not expected that the measured winds (had they been available) would yield any different result, as they are not expected to show significant variations from one day to another during magnetically quiet times, to which the data in this study corresponds.

To further confirm the role of electrodynamic influence on the daytime airglow intensities, we have investigated the ionospheric behaviour at two different locations, Trivandrum (magnetic equatorial location) and Ahmedabad (typically the northern crest location of the EIA). These independent

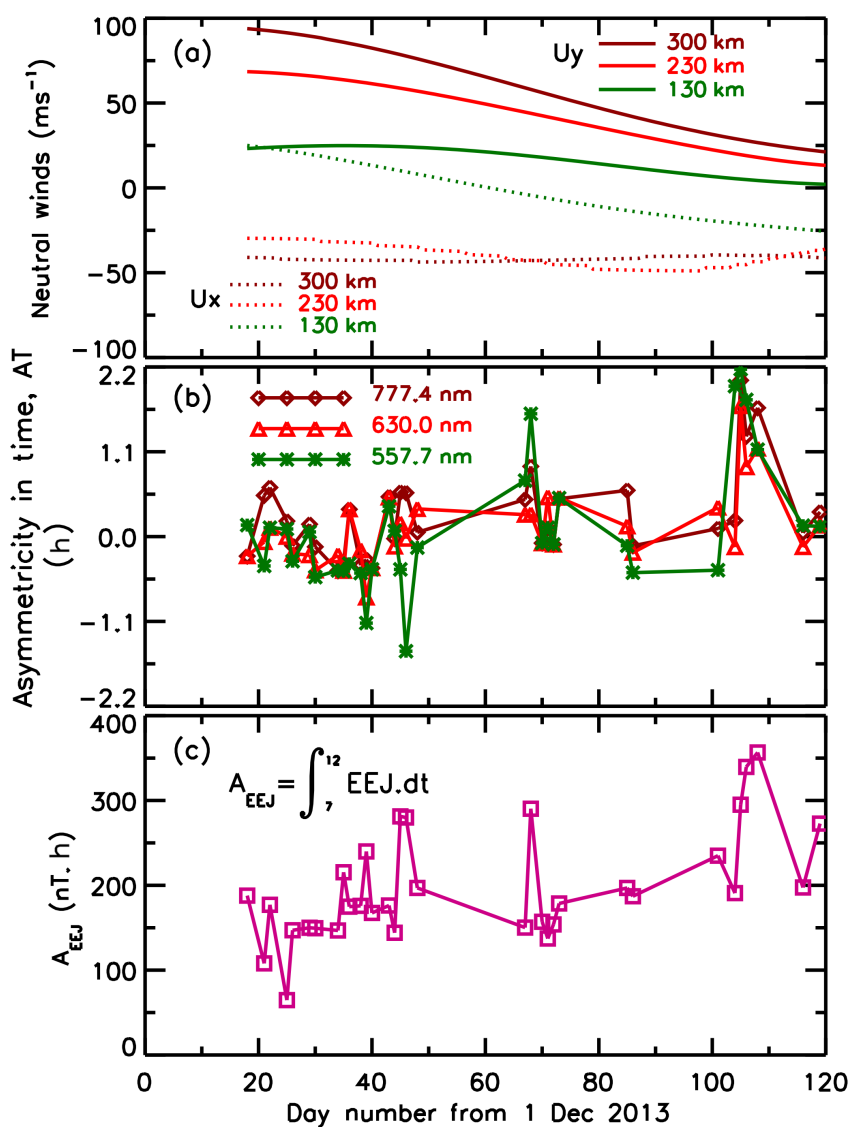

Figure 5. The variation in (a) neutral winds at the three different emission altitudes, (b) the asymmetricity in time (AT) at the three dayglow emission intensities on different days, and (c) EEJ strength integrated over 07:00-12:00 LT ( $\left.A_{\mathrm{EEJ}}\right)$ on the days corresponding to optical data are shown. The $x$ axis shows the day number starting from 1 December 2013.

ionospheric measurements were segregated into two categories: those corresponding to the days when all the dayglow emission intensities showed symmetric diurnal behaviour (AT $\leq 0.4 \mathrm{~h}$ ) and those that showed asymmetric diurnal behaviour (AT $>0.4 \mathrm{~h}$ ). Figure $6 \mathrm{a}$ and $\mathrm{b}$ show the peak $\mathrm{F}$ region height $(h m \mathrm{~F} 2)$ over Trivandrum on the days with symmetric and asymmetric intensity distribution in dayglow emission intensities, respectively (ionosonde data corresponding to the days with symmetric diurnal behaviour in optical emission intensities are not available from Trivandrum during December 2013). The variation in the values of $h m \mathrm{~F} 2$ is considered to be representative of the $\mathrm{F}$ region electric field over the dip equator. It can be seen in Fig. 6a that the peak $h m \mathrm{~F} 2$ decreases in the afternoon on the days with symmetric diurnal behaviour. However, on the days with asymmetric diurnal behaviour (Fig. 6b), the $h m \mathrm{~F} 2$ shows an increasing trend, indicating that the equatorial electrodynamics are active in the afternoon. Figure $6 \mathrm{c}$ and d show the ionospheric electron content (IEC) obtained from the digisonde mea- 

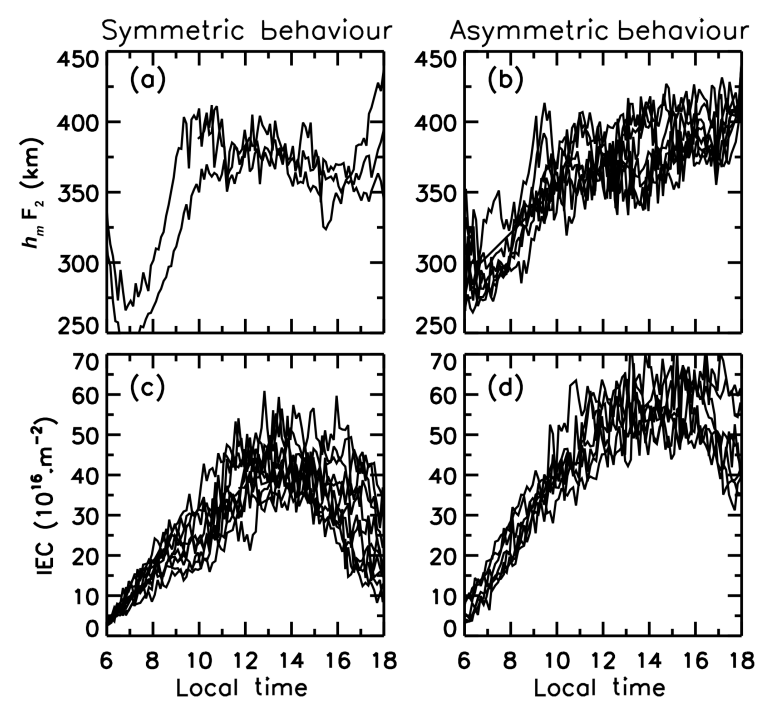

Figure 6. Variation of peak height of F2 layer over Trivandrum (equatorial station) and ionospheric electron content (IEC) over Ahmedabad (station typically under the northern crest of the EIA) on the days with (a, c) symmetric and (b, d) asymmetric diurnal dayglow intensity behaviour, respectively, are depicted.

surements over Ahmedabad on these days. It is very clear from these figures that the electron density over Ahmedabad peaked at around 14:00 LT on the days with symmetric diurnal behaviour, whereas on the days with asymmetric diurnal behaviour the electron density keeps increasing and its peak occurs later than 15:00 LT. It also indicates that the electrodynamics had been active in the late afternoon hours on the days with an asymmetric diurnal pattern observed in the dayglow emission intensities. Further, the IEC values are of greater magnitudes on the days with asymmetric diurnal behaviour (Fig. 6d) as compared to the days with symmetric diurnal behaviour (Fig. 6c). It is striking to note that the optical dayglow measurements obtained over Hyderabad (a location between Trivandrum and Ahmedabad; Fig. 1) show peak emission intensities at around 13:00 LT on the days with asymmetric diurnal behaviour in comparison to 15:00 LT in IEC over Ahmedabad, which can be attributed to the movement of the crest of the EIA. These independent measurements add credence to our interpretation that the temporal behaviour seen in the optical neutral dayglow emission intensities are governed by electrodynamic forces that originate at the magnetic equator.

\section{Discussion}

The nighttime airglow intensities vary purely as a function of densities of reactants, and their behaviour does not show any set pattern, whereas the daytime emission intensities show a broad solar zenith-angle-dependent variation primarily due to the solar control of several of the produc- tion mechanisms. However, it is quite interesting to note the clear changes that are brought in in the diurnal intensity variation of neutral daytime airglow emission intensities. For OI $630.0 \mathrm{~nm}$ dayglow emission, the data obtained during 2001 from a low-latitude location, Carmen Alto, in Chile (Pallamraju and Chakrabarti, 2006) showed such asymmetric diurnal behaviour as seen in the present study. However, later in a low solar activity epoch such an asymmetric diurnal pattern in the dayglow emission intensities was not noted. In Fig. 7 we reproduce Fig. 1 from Laskar et al. (2015), where the data that were obtained from Hyderabad, India, over the years of 2011-2013 are shown. The diurnal behaviour in intensities in all these years shows a broadly symmetric nature as seen in Fig. 3a, b, and c of the present study. The optical data obtained from that epoch did not show any asymmetric diurnal behaviour in the emission intensities. It is important to note that for the days of optical data that exist in the years 2001, 2011, 2012, 2013, and 2014, the average sunspots numbers were $160,35,52,53$, and 144 , respectively, and so there seems to be a solar activity dependence in the observed AT values in optical dayglow emissions.

In order to characterize the solar activity effect, we have looked at the $630.0 \mathrm{~nm}$ optical dayglow emission intensity pattern as presented in the literature at different times and locations. The $630.0 \mathrm{~nm}$ dayglow emission is chosen due to the availability of a large set of observations at this emission (for over 25 years, although not continuous) in the published literature. The AT values were calculated for each day, and their mean values in different years are shown in Fig. 8. The $x$ axis shows the year, and the $y$ axis (on the left) shows the mean AT values (red dots) (the relevant literature which has been considered for these data is shown in the figure). We have also plotted (on the right side of the $y$ axis) the monthly average sunspot numbers (dark dots). Observations by Laskar et al. (2015) during 2011-2013 show a symmetric diurnal pattern at all three wavelength emissions and in the absence of any possibility to calculate the AT values for these days (as the emission peak occurs around noontime; however, as the solar glare enters directly over the slit, no data were obtainable), the values of AT are approximated to zero during these years. It should be mentioned here that in the present experimental setup, the slit of MISE is oriented in the zonal direction (for the study of longitudinal variations) due to which the direct entry of solar glare during noontime is avoided, enabling us to obtain continuous dayglow data throughout the day without any gap. Also, some of the earlier results published in the literature that are used in this study to estimate the AT values plotted in Fig. 8 had a smaller field of view of $\sim 4^{\circ}$ (Sridharan et al., 1999) because of which direct entry of solar glare was not an issue. The mean AT values calculated from the present observations at the three emission wavelengths are shown in different colours. It is striking to note that the variations of AT values go almost hand-in-hand with those in sunspot numbers. This clearly shows that the asymmetricity in time observed in optical neutral dayglow 

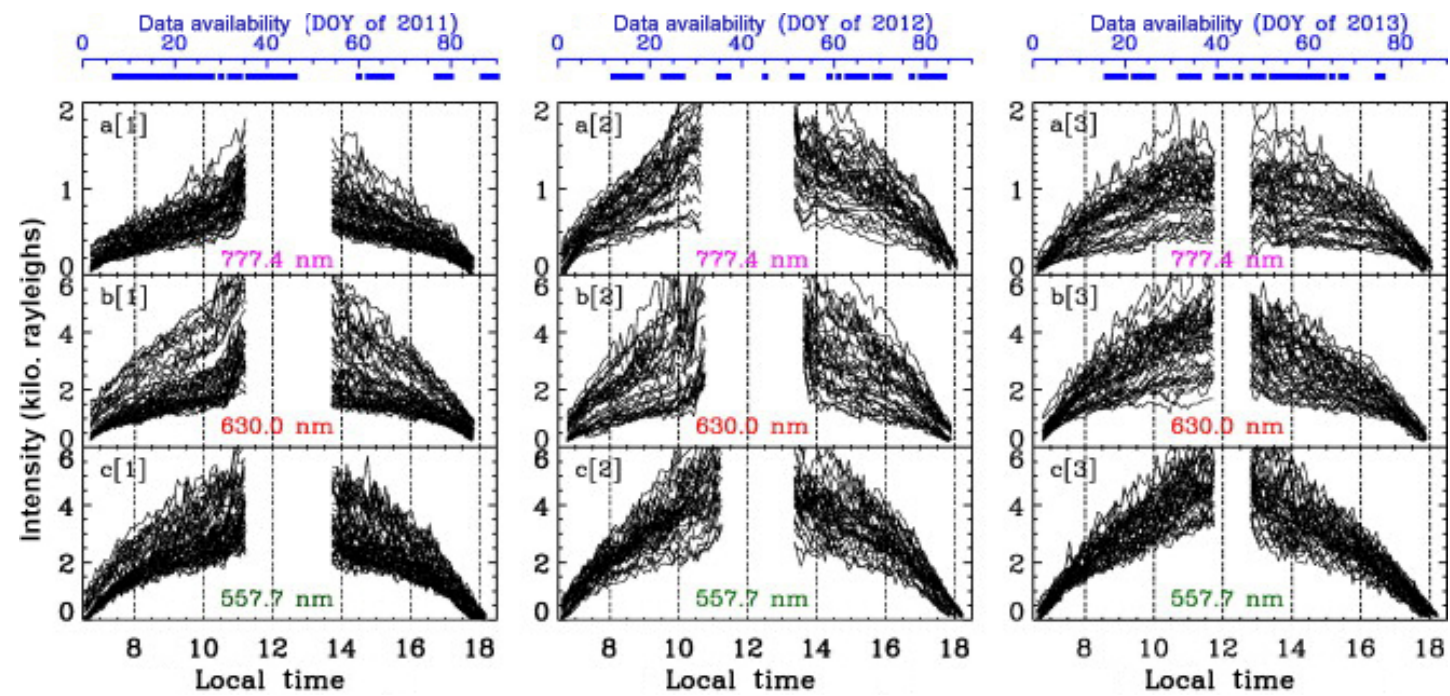

Figure 7. Reproduced from Fig. 1 of Laskar et al. (2015). The diurnal variations of the dayglow emission intensities for the 3 years $2011-$ 2013 (left to right) and for the three wavelengths (top to bottom) are shown. The additional axes at the top represent the day of the year (DOY) on which dayglow data are available. It can be noted that the diurnal intensity pattern of all the emissions show a symmetric, broad solar zenith angle dependence in these years unlike the ones reported in the present data from the year 2014, in which deviations from solar zenith angle dependence seem to exit (Fig. 3d, e, f).

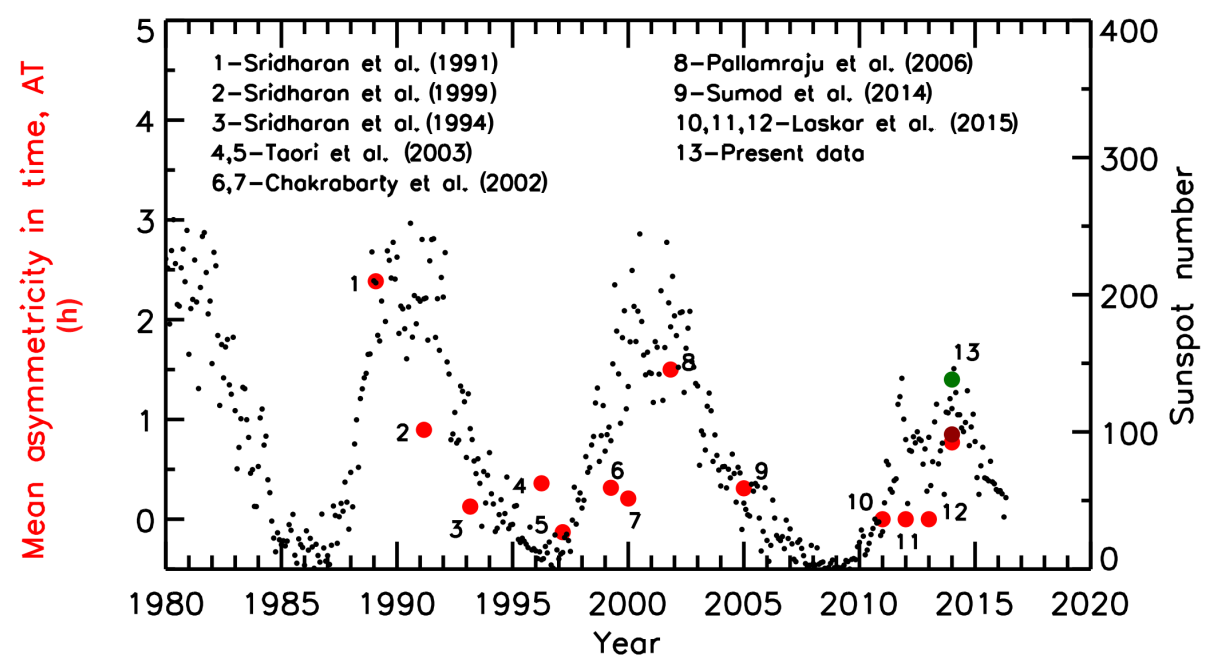

Figure 8. Variation of mean asymmetricity in time (AT) at OI $630.0 \mathrm{~nm}$ emissions in different years as obtained from the published literature is shown. The monthly averaged sunspot number is also plotted. A striking similarity between them indicates that the equatorial electric field has a direct role in the observed diurnal behaviour of the neutral optical dayglow emission intensities.

emissions has a solar activity dependence. This is interpreted to be due to the increase in equatorial electric field strengths with solar activity.

It thus shows the dominance of electrodynamic processes over the photochemical processes in bringing about temporal variations in the neutral dayglow emissions and is also a good example of $\mathrm{E}$ and $\mathrm{F}$ region coupling in the equatorialand low-latitude ionosphere-thermosphere system. A consequence of this effect is shown in Fig. 6, where a movement of $\mathrm{F}$ region height in the afternoon to higher altitudes on some days over Trivandrum (equatorial station) (Fig. 6b) and a corresponding increase in the IEC over Ahmedabad (station near the northern crest of the EIA) is seen (Fig. 6d). The strength of this phenomenon varies with respect to local time, season, and solar activity. In the low solar activity period (2011-2013), the electric field strengths are smaller (Fejer and Scherliess, 1995) and apparently not sufficient to move the ionization to regions far away from the magnetic equator. Hence, the dayglow emission intensities measured from Hyderabad showed a symmetric photochemical emission be- 
haviour in their respective intensities. This can also be seen in the present study wherein the negative AT values on some days correlate with smaller integrated electrojet strengths (Fig. $5 \mathrm{~b}$ and c) and, hence, weaker electrodynamics (Fig. 6a and c). Further, the results from midlatitudes (Boston), where equatorial electrodynamic effects do not exist, do show a symmetric type of behaviour in OI $630.0 \mathrm{~nm}$ diurnal emission intensities (Pallamraju and Chakrabarti, 2006) as seen in the present study with weaker electrodynamics. These issues add credence to the inference regarding the influence of an electrodynamic effect in the neutral daytime airglow emissions.

To appreciate this issue further, attention is drawn to another study wherein the dayglow OI $630.0 \mathrm{~nm}$ emissions were measured from the magnetic equatorial station Thumba in India. It was seen that the shape of the diurnal pattern of the $630.0 \mathrm{~nm}$ dayglow was similar to that of the EEJ with a time shift (Sridharan et al., 1999). This time gap had been interpreted to be the time taken for the plasma to move from the $\mathrm{E}$ to $\mathrm{F}$ region under the influence of $\boldsymbol{E} \times \boldsymbol{B}$ drifts. The fact that it was indeed so was also confirmed by the simultaneously operating VHF doppler radar, where the $\boldsymbol{E} \times \boldsymbol{B}$ drifts obtained by the measured eastward electric fields were consistent with the observed time gaps between the EEJ and $630.0 \mathrm{~nm}$ dayglow variation. This result indicated the imprint of electrodynamic effect on the $630.0 \mathrm{~nm}$ dayglow emission intensities, both of which were obtained from the same location. Observations from an EIA crest region, $\mathrm{Mt}$ Abu $\left(24.6^{\circ} \mathrm{N}, 72.8^{\circ} \mathrm{E}\right)$ in India, during high solar activity showed different behaviour in the OI $630.0 \mathrm{~nm}$ emission intensities on the equatorial electrojet and counter electrojet days (Chakrabarty et al., 2002). All these results corroborate our conclusion that the asymmetric diurnal behaviour of the optical dayglow emission intensities seen in the low latitudes is mainly due to the equatorial electrodynamic variations. However, the effect of neutral winds on the dayglow emissions cannot be ruled out completely. Ultimately, a comprehensive model is needed to fully understand the complex coupled behaviour of the thermosphere/ionosphere and the underlying processes at equatorial and off-equatorial lowlatitude regions.

In this study it is shown that not only the OI $630.0 \mathrm{~nm}$ dayglow intensity variations but also those at OI $777.4 \mathrm{~nm}$ and OI $557.7 \mathrm{~nm}$ show asymmetric diurnal behaviour. It should be remembered that the peak dayglow emission intensities at any of the wavelengths are not correlated with EEJ strengths; they are related with the asymmetricity in time, indicating that it is not the total emission intensities but their temporal variability that is governed by the EEJ strength.

\section{Conclusion}

Oxygen-neutral dayglow emissions at multiple wavelengths measured during 2013-2014 showed that the emissions' di- urnal behaviour in intensities was both symmetric and asymmetric with respect to local noon. While the symmetric diurnal behaviour can be understood in terms of solar zenith angle variation of the production mechanisms, the cause of asymmetric behaviour in diurnal emission intensities is not apparent. Against this background, its possible causes have been investigated in terms of neutral winds and equatorial electrodynamics. Using the equatorial electrojet strength data and ionospheric behaviour on all these days, it has been conclusively shown that the equatorial electrodynamics that are operative on a given day give rise to the observed asymmetric diurnal behaviour in the neutral oxygen dayglow emission variability. This aspect has been discussed in a wider context. It has been noted that in the low solar activity period, the diurnal variability in the oxygen emission intensities was predominantly symmetric with respect to local noon, while they were asymmetric during high solar activity periods. This again gives a broader picture to the ionosphere-thermosphere systemic behaviour as the neutral dayglow emission intensities are sensitive to the electrodynamic changes that happen over a solar cycle.

\section{Data availability}

For this study, the optical dayglow data from Hyderabad and ionospheric data from Ahmedabad were obtained by the Physical Research Laboratory and can be made available on request. The EEJ data can be obtained from the Indian Institute of Geomagnetism. The ionosonde data from Trivandrum can be obtained from the Space Physics Laboratory. The sunspot number data are obtained from the daily solar dataset maintained by NOAA (ftp://ftp.swpc.noaa.gov/pub/indices).

Acknowledgements. The authors acknowledge the assistance provided by Abdul Moid, Sambasivam Rao, and Abhilash Maryada in the operation of MISE at Jawaharlal Nehru Technological University, Hyderabad, India. This work is supported by the Department of Space, Government of India.

The topical editor, J. Makela, thanks the three anonymous referees for help in evaluating this paper.

\section{References}

Chakrabarty, D., Sekar, R., Chandra, H., Narayanan, R., Pathan, B. M., and Subbarao, K. S. V.: Characterizations of the diurnal shapes of OI $630.0 \mathrm{~nm}$ dayglow intensity variations: inferences, Ann. Geophys., 20, 1851-1855, doi:10.5194/angeo-201851-2002, 2002.

Chakrabarti, S., Pallamraju, D., Baumgardner, J., and Vaillancourt, J.: HiTIES: a high throughput imaging echelle spectrograph for ground-based visible airglow and auroral studies, J. Geophys. Res., 106, 30337-30348, 2001. 
Das, U., Pallamraju, D., and Chakrabarti, S.: Effect of an X-Class solar flare on the OI $630 \mathrm{~nm}$ dayglow emissions, J. Geophys. Res., 115, A08302, doi:10.1029/2010JA015370, 2010.

Drob, D. P., Emmert, J. T., Meriwether, J. W., Makela, J. J., Doornbos, E., Conde, M., Hernandez, G., Noto, J., Zawdie, K. A., McDonald, S. E., Huba, J. D., and Klenzing, J. H.: An update to the Horizontal Wind Model (HWM): The quiet time thermosphere, Earth and Space Science, 2, 301-319, doi:10.1002/2014EA000089, 2015.

Emmert, J. T., Richmond, A. D., and Drob D. P.: A computationally compact representation of Magnetic-Apex and Quasi-Dipole coordinates with smooth base vectors, J. Geophys. Res., 115, A08322, doi:10.1029/2010JA015326, 2010.

Fejer, B. G. and Scherliess, L.: Time dependent response of equatorial ionospheric electric fields to magnetospheric disturbances, Geophys. Res. Lett., 22, 851-854, doi:10.1029/95GL00390, 1995.

Gerrard, A. J. and Meriwether, J. W.: Initial daytime and nighttime SOFDI observations of thermospheric winds from FabryPerot Doppler shift measurements of the 630-nm OI line-shape profile, Ann. Geophys., 29, 1529-1536, doi:10.5194/angeo-291529-2011, 2011.

Hays, P. B., Rusch, D. W., Roble, R. G., and Walker, J. C. G.: The OI (6300 A) airglow, Rev. Geophys., 16, 225-232, 1978.

Laskar, F. I., Pallamraju, D., Lakshmi, T. V., Reddy, M. A., Pathan, B. M., and Chakrabarti, S.: Investigations on vertical coupling of atmospheric regions using combined multiwavelength optical dayglow, magnetic, and radio measurements, J. Geophys. Res.Space, 118, 4618-4627, doi:10.1002/jgra.50426, 2013.

Laskar, F. I., Pallamraju, D., Veenadhari, B., Lakshmi, T. V., Reddy, M. A., and Chakrabarti, S.: Gravity waves in the thermosphere: Solar activity dependence, Adv. Space. Res., 55, 1651-1659, 2015.

Maharaj-Sharma, R. and Shepherd, G. G.: Solar variability of the day-time atomic oxygen $\mathrm{O}\left({ }^{1} \mathrm{~S}\right)$ emission in the middle and lower thermosphere, J. Geophys. Res., 109, A03303, doi:10.1029/2003JA010183, 2004.

Makela, J. J., Fisher, D. J., Meriwether, J. W., Buriti, R. A., and Medeiros, A. F.: Near-continual ground-based nighttime observations of thermospheric neutral winds and temperatures over equatorial Brazil from 2009 to 2012, J. Atmos. Sol.-Terr. Phy., 103, 94-102, 2013.

Moffett, R. J.: The equatorial anomaly in the electron distribution of the Terrestrial F region, Fund. Cosmic. Phys., 4, 313-387, 1979.

Nakamura, T., Tsuda, T., Miyagawa, H., Matsushita, Y., Fukunishi, H., Takahashi, Y., and Yamada, Y.: Propagation directions of gravity wave patterns observed in $\mathrm{OH}$ CCD images during SEEK campaign, Geophys. Res. Lett., 25, 1793-1796, 1998.

Narayanan, R., Desai, J. N., Modi, N. K., Raghavarao, R., and Sridharan, R.: Dayglow photometry: a new approach, Appl. Optics, 28, 2138-2142, 1989.

Pallam Raju, D., Sridharan, R., Gurubaran, S., and Raghavarao, R.: First results from ground-based daytime optical investigation of the development of the equatorial ionization anomaly, Ann. Geophys., 14, 238-245, doi:10.1007/s00585-996-0238-9, 1996.

Pallamraju, D., Baumgardner, J., and Chakrabarti, S.: A multiwavelength investigation of the Ring effect in the day sky spectrum, Geophys. Res. Lett., 27, 1875-1878, 2000.
Pallamraju, D., Baumgardner, J., and Chakrabarti, S.: HIRISE: A ground-based high-resolution imaging spectrograph using echelle grating for measuring daytime airglow/auroral emissions, J. Atmos. Sol.-Terr. Phy., 64, 1581-1587, 2002.

Pallamraju, D., Chakrabarti, S., Doe, R., and Pederson, T.: First ground-based OI $630 \mathrm{~nm}$ optical measurements of daytime cusplike and F-region auroral precipitation, Geophys. Res. Lett., 31, L08807, doi:10.1029/2003GL019173, 2004.

Pallamraju, D. and Chakrabarti, S.: First ground-based measurements of OI 6300 A daytime aurora over Boston in response to the 30 October 2003 geomagnetic storm, Geophys. Res. Lett., 32, L03S10, doi:10.1029/2004GL021417, 2005.

Pallamraju, D. and Chakrabarti, S.: Contributions of Imaging Echelle spectrographs to daytime optical aeronomy, J. Atmos. Sol.-Terr. Phy., 68, 1459-1471, 2006.

Pallamraju, D., Das, U., and Chakrabarti, S.: Short- and longtimescale thermospheric variability as observed from OI $630.0 \mathrm{~nm}$ dayglow emissions from low latitudes, J. Geophys. Res.-Space, 115, A06312, doi:10.1029/2009JA015042, 2010.

Pallamraju, D., Laskar, F. I., Singh, R. P., Baumgardner, J., and Chakrabarti S.: MISE: A multiwavelength imaging spectrograph using echelle grating for daytime optical aeronomy investigations, J. Atmos. Sol.-Terr. Phy., 103, 176-183, 2013.

Pallamraju, D., Karan, D. K., and Phadke, K. A.: First three dimensional wave characteristics in the daytime upper atmosphere derived from ground-based multiwavelength oxygen dayglow emission measurements, Geophys. Res. Lett., 43, 5545-5553, doi:10.1002/2016GL069074, 2016.

Raghavarao, R., Sharma, P., and Sivaraman, M. R.: Correlation of Ionization anomaly with the intensity of the electrojet, Space Res., XVIII, 277-280, 1978.

Raghavarao, R., Hoegy, W. R., Spencer, N. W., and Wharton, L. E.: Neutral temperature anomaly in the equatorial thermosphere A source of vertical winds, Geophys. Res. Lett., 20, 1023-1026, 1993.

Shepherd, G. G., Siddiqi, N. J., Wiens, R. H., and Zang, S.: Airglow measurements of possible changes in the ionosphere and middle atmosphere, Adv. Space Res., 20, 2127-2135, 1997.

Shiokawa, K., Otsuka, Y., and Ogawa, T.: Propagation characteristics of nighttime mesospheric and thermospheric waves observed by optical mesosphere thermosphere imagers at middle and low latitudes, Earth Planets Space, 61, 479-491, 2009.

Singh, R. P. and Pallamraju, D.: On the latitudinal distribution of mesospheric temperatures during sudden stratospheric warming events, J. Geophys. Res.-Space, 120, 2926-2939, doi:10.1002/2014JA020355, 2015.

Singh, R. P. and Pallamraju, D.: Effect of cyclone Nilofar on mesospheric wave dynamics as inferred from optical nightglow observations from Mt. Abu, India, J. Geophys. Res.-Space, 121, 58565867, doi:10.1002/2016JA022412, 2016.

Singh, V., McDade, I. C., Shepherd, G. G., Solheim, B. H., and Ward, W. E.: The O(1D) dayglow emission as observed by the wind imaging interferometer on UARS, Adv. Space Res., 17, 1114, 1996.

Solomon, S. C. and Abreu, V.: The 630.0nm dayglow, J. Geophys. Res., 94, 6817-6824, 1989.

Sridharan, R., Raghavarao, R., Gurubaran, S., and Narayanan, R.: First results of OI $630.0 \mathrm{~nm}$ dayglow measurements from equatorial latitudes, J. Atmos. Sol.-Terr. Phy., 53, 521-528, 1991. 
Sridharan, R., Haider, S. A., Gurubaran, S., Sekar, R., and Narayanan, R.: OI 630.0-nm dayglow in the region of equatorial ionization anomaly: Temporal variability and its causative mechanism, J. Geophys. Res., 97, 13715-13721, 1992.

Sridharan, R., Sekar, R., and Gurubaran, S.: Two dimensional high resolution imaging of the equatorial plasma fountain, J. Atmos. Sol.-Terr. Phy., 55, 1661-1665, 1993a.

Sridharan, R., Narayanan, R., Modi, N. K., and Pallamraju, D.: A novel mask design for multiwavelength dayglow photometry, Appl. Optics, 32, 4178-4180, 1993b.

Sridharan, R., Pallamraju, D., Raghavarao, R., and Ramarao, P. V. S.: Precursor to equatorial spread-F in 0I $630.0 \mathrm{~nm}$ dayglow, Geophys. Res. Lett., 21, 2797-2800, 1994.

Sridharan, R., Modi, N. K., Pallam Raju, D., Narayanan, R., Pant, T. K., Taori, A., and Chakrabarty, D.: A Multiwavelength daytime photometer - a new tool for the investigation of atmospheric processes, Meas. Sci. Technol., 9, 585-591, 1998.

Sridharan, R., Pallamraju, D., Somayajulu, V. V., Taori, A., Chakrabarty, D., and Raghavarao, R.: Imprint of equatorial electrodynamical processes in the OI $630.0 \mathrm{~nm}$ dayglow, J. Atmos. Sol.-Terr. Phy., 61, 1143-1155, 1999.

Sumod, S. G., Pant, T. K., Vineeth, C., and Hossain, M. M.: On the ionospheric and thermospheric response of solar flare events of 19 January 2005: An investigation using radio and optical techniques, J. Geophys. Res.-Space, 119, 5049-5059, doi:10.1002/2013JA019714, 2014.

Taori, A., Sridharan, R., Chakrabarty, D., Modi, N. K., and Narayanan, R.: Significant upper thermospheric contribution to the $\mathrm{O}\left({ }^{1} \mathrm{~S}\right) 557.7 \mathrm{~nm}$ dayglow emission: first ground based evidence, J. Atmos. Sol.-Terr. Phy., 65, 121-128, 2003.
Taylor, M. J., Bishop, M. B., and Taylor, V.: All-sky measurements of short period waves imaged in the OI $(557.7 \mathrm{~nm}), \mathrm{Na}$ $(589.2 \mathrm{~nm})$ and near infrared $\mathrm{OH}$ and $\mathrm{O}_{2}(0,1)$ nightglow emissions during the ALOHA-93 campaign, Geophys. Res. Lett., 22, 2833-2836, 1995.

Tinsley, B. A., Christensen, A. B., Bittencourt, J., Gouveia, H., Angreji, P. D., and Takahashi, H.: Excitation of oxygen permitted line emissions in the tropical nightglow, J. Geophys. Res., 78, 1174-1186, 1973.

Tyagi, S. and Singh, V.: The morphology of oxygen greenline dayglow emission, Ann. Geophys., 16, 1599-1606, doi:10.1007/s00585-998-1599-z, 1998.

Upadhayaya, A. K. and Singh, V.: Effects of temperature dependence of reaction $\mathrm{N}_{2}\left(\mathrm{~A}^{3} \mathrm{~S}_{\mathrm{u}}^{+}\right)+\mathrm{O}$ ongreenline dayglow emission, Ann. Geophys., 20, 2039-2045, doi:10.5194/angeo-202039-2002, 2002.

Wallace, L. and McElroy, M. B.: The visual dayglow, Planet. Space Sci., 14, 677-708, 1966.

Witasse, O., Lilensten, J., Lathuillere, C., and Blelly, P. L.: Modeling the OI 630.0 and $557.7 \mathrm{~nm}$ thermospheric dayglow during EISCAT-WINDII coordinated measurements, J. Geophys. Res., 104, 24639-24655, 1999.

Zhang, S. P. and Shepherd, G. G.: Solar influence on the $\mathrm{O}(1 \mathrm{D})$ dayglow emission rate: Global scale measurements by WINDII on UARS, Geophys. Res. Lett., 31, L07804, doi:10.1029/2004GL019447, 2004.

Zhang, S. P. and Shepherd, G. G.: On the response of the O(1S) dayglow emission rate to the Sun's energy input: An empirical model deduced from WINDII/UARS global measurements, J. Geophys. Res., 110, A03304, doi:10.1029/2004JA010887, 2005. 